\title{
Environmental Risk Assessment of Genetically Modified Organisms by a Fuzzy Decision Support System
}

\author{
Francesco Camastra $^{1}$, Angelo Ciaramella ${ }^{1}$, Valeria Giovannelli ${ }^{2}$, \\ Matteo Lener ${ }^{2}$, Valentina Rastelli ${ }^{2}$, Antonino Staiano ${ }^{1}$, \\ Giovanni Staiano ${ }^{2}$, and Alfredo Starace ${ }^{1}$ \\ 1 Dept. of Science and Technology, University of Naples Parthenope, \\ Centro Direzionale Isola C4, I-80143, Napoli, Italy \\ \{camastra, angelo.ciaramella, staiano\}@ieee.org, alfredo.starace@gmail.com \\ 2 Nature Protection Dept., Institute for Environmental Protection and Research \\ (ISPRA), via v. Brancati 48, 00144 Roma \\ \{valeria.giovannelli, matteo.lener, valentina.rastelli, \\ giovanni.staiano\}@isprambiente.it
}

\begin{abstract}
Aim of the paper is the development of a Fuzzy Decision Support System (FDSS) for the Environmental Risk Assessment (ERA) of the deliberate release of genetically modified plants. The evaluation process permits identifying potential impacts that can achieve one or more receptors through a set of migration paths. ERA process is often performed in presence of incomplete and imprecise data and is generally yielded using the personal experience and knowledge of the human experts. Therefore the risk assessment in the FDSS is obtained by using a Fuzzy Inference System (FIS), performed using jFuzzyLogic library. The decisions derived by FDSS have been validated on real world cases by the human experts that are in charge of ERA. They have confirmed the reliability of the fuzzy support system decisions.
\end{abstract}

Keywords: Fuzzy Support Decision Systems, Risk Assessment, Genetically Modified Organisms, Fuzzy Control Language, jFuzzyLogic library.

\section{Introduction}

The development of genetic engineering in the last years produced a very high number of genetically modified organisms (GMOs). Whereas in USA the use of GMOs is widely spread in agriculture, in Europe there are discordant policies w.r.t. GMOs. For instance, commercialization of food and feed containing or consisting of GMOs is duly approved in European Community (EC), while cultivation of new genetically modified crops are not adopted. The maize MON 810, approved by the old EC legislation framework, is currently the unique GMO cultivated in the EC (e.g., Czech Republic, Poland, Spain, Portugal, Romania and Slovakia). According to EC, the environmental release of GMOs is ruled by Directive 200118EC and Regulation 18292003EC. The Directive refers to the

A. Petrosino, L. Maddalena, P. Pala (Eds.): ICIAP 2013 Workshops, LNCS 8158, pp. 428-435, 2013.

(C) Springer-Verlag Berlin Heidelberg 2013 
deliberate release into the environment of GMOs and sets out two regulatory regimes: Part $\mathrm{C}$ for the placing on the market and Part $\mathrm{B}$ for the deliberate release for any other purpose, i.e. field trials [16]. In both legislations the notifier, i.e., the person who requests the release into the environment of GMO, must perform an Environmental Risk Assessment (ERA) on the issue. The ERA is formally defined as "the evaluation of risks to human health and the environment, whether direct or indirect, immediate or delayed, which the deliberate release or the placing on the market of GMOs may pose". ERA should be carried out case by case, meaning that its conclusion may depends on the GM plants and trait concerned, their intended uses, and the potential receiving environments. The ERA process should lead to the identification and evaluation of potential adverse effects of the GMO, and, at the same time, it should be conducted with a view for identifying if there is a need for risk management and it should provides the basis for the monitoring plans. The aim of this work is the development of a decision system that should advise and help the notifier in performing the ERA about the cultivation of a specific genetically modified plant (GMP). ERA process is often performed in presence of incomplete and imprecise data. Moreover, it is generally yielded using the personal experience and knowledge of the notifier. Therefore the usage of fuzzy reasoning in the ERA support decision system is particularly appropriate as witnessed by the extensive application of

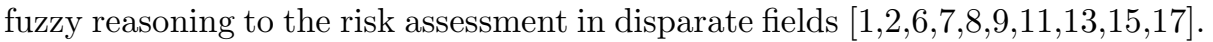
Having said that, the fuzzy support decision system, object of the paper, is inspired by the methodological proposal of performing ERA on GMP field trials [16. The methodology would allow to describe the relationships between potential receptors and the harmful characteristics of a GMP field trial, leading to the identification of potential impacts. The paper is organized as follows: In Section 2 the methodological proposal that has inspired the system is described; The FDSS structure of the Fuzzy System is discussed in Section 3 Section 4 describes how the system validation has been performed; Finally some conclusions are drawn in Section 5 .

\section{The Methodological Approach}

The methodological proposal, that has inspired the system object of the paper, is based on a conceptual model [16]. The schema, shown in Figure1, illustrates the possible paths of the impact from a specific source to a given receptor through disparate diffusion factors and migration routes. The model implies that the notifier fills an electronic questionnaire. The notifier answers are collected in a relational database management system and, in a second time, become input of a fuzzy decision support engine that is the system core and provides to the notifier the overall evaluation of risk assessment related to a specific GM plant. The questionnaire can be grouped in specific sets of questions where each set corresponds to a specific box of the diagram of the conceptual model. For each block the potential effects are calculated by using fuzzy concepts and a fuzzy reasoning system. The questions can be of two different types, e.g., qualitative 


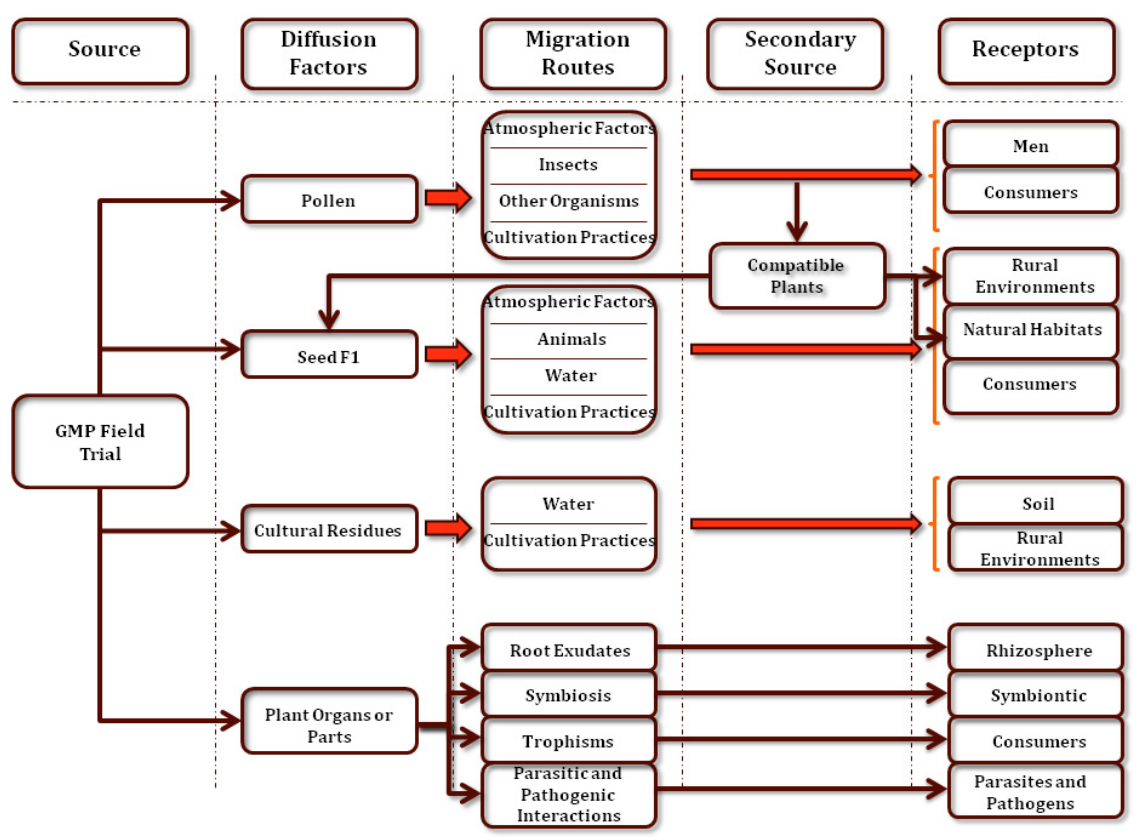

Fig. 1. Conceptual model

and quantitative. The former is typically descriptive and it is not used by fuzzy decision support system in the reasoning process. On the contrary, the latter is used by the fuzzy engine and can be an item chosen within a limited number of possible replies or a numeric or a boolean value.

\section{The Fuzzy Decision Support System}

The FDSS, object of the paper, has the same architecture of a Fuzzy Logic Control System. Moreover, the Fuzzy Inference System (FIS) of FDSS has been implemented using the jFuzzyLogic library. Therefore the section is organized in two subsections. In the former subsection we discuss the architecture of a generic Fuzzy Logic Control System and, hence, of our system. In the latter subsection jFuzzyLogic is described, in detail.

\subsection{A Fuzzy Logic Control System}

A Fuzzy Logic Control (FLC) system incorporates the knowledge and experience of a human operator, the so-called expert, in the design of a system that controls a process whose input-output relationships are described by a set of fuzzy control rules, e.g., IF-THEN rules. We recall that the antecedent is the part of rule delimited by the keywords IF and THEN. Whereas the consequent is the part of 


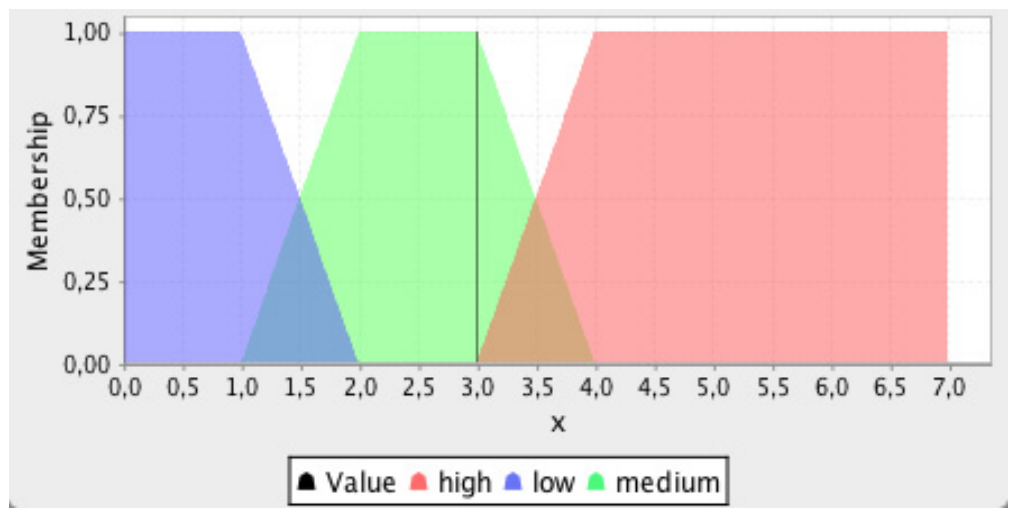

Fig. 2. Membership functions of the linguistic variable number of insert copies

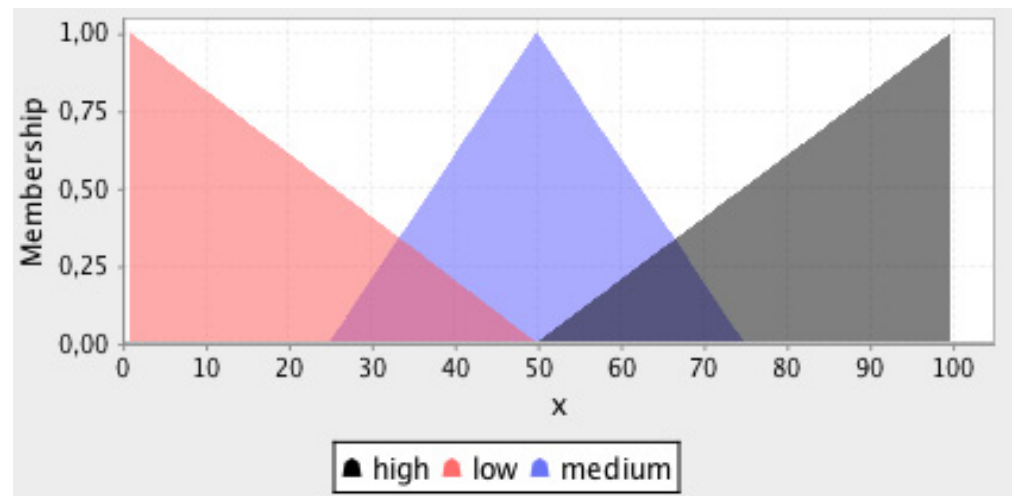

Fig. 3. Membership functions of the linguistic variable potential risk of the insert

the rule that follows the keyword THEN. The rules involve linguistic variables $(L V \mathrm{~s})$ that express qualitative high level concepts. A typical FLC architecture is composed of four principal components: a fuzzifier, a fuzzy rule base, an inference engine and a defuzzifier [12. The fuzzifier has the task of transforming crisp measured data (e.g., number of insert copies is 1) into suitable linguistic values, namely the data becomes, for instance, number of insert copies is low. The fuzzy rule base stores the operation knowledge of the process of the domain experts. The inference engine is the FLC core, and it can simulate human decision making process by performing approximate reasoning in order to achieve a desired control strategy. The defuzzifier is used for yielding a control action inferred by the inference engine. In the inference engine the generalized modus 
ponens 12 plays an important role. For the application of fuzzy reasoning in FLCs, the generalized modus ponens can written as

Premise $1:$ IF $x$ is $A$ THEN $y$ is $B$
Premise $2: x$ is $A^{\prime}$
Conclusion $y$ is $B^{\prime}$

where $A, A^{\prime}, B$ and $B^{\prime}$ are fuzzy predicates i.e., Fuzzy Sets or Fuzzy Relations. In general, a fuzzy control rule, e.g., premise 1 , is a fuzzy relation expressed by a fuzzy implication. In most general cases, the fuzzy rule bases has the form of a Multi-Input-Multi-Output (MIMO) system. In this case the inference rules are combined by using the connectives AND and ELSE that can be interpreted as the intersection and the union for different definitions of fuzzy implications, respectively. For instance, if we consider the LV number of insert copies as shown in Figure 2, the fuzzy inference system of the LVs number of insert copies and number of introduced sequences could be represented by:

\author{
IF number of insert copies is Low AND \\ number of introduced sequences is High THEN \\ potential risk of the insert is High \\ ELSE \\ IF number of insert copies is High AND \\ number of introduced sequences is Medium THEN \\ potential risk of the insert is High \\ ELSE \\ IF number of insert copies is Low AND \\ number of introduced sequences is Low THEN \\ potential risk of the insert is Low
}

In Figure 3 an example of fuzzy memberships for the output LV potential risk of the insert is presented. Finally, a crisp (i.e., non fuzzy) output is obtained considering a Center of Area defuzzifier 12.

\title{
3.2 jFuzzyLogic
}

jFuzzyLogic 4] is an open source software library for fuzzy systems which allows to design Fuzzy Logic Controllers supporting the standard Fuzzy Control Programming [10], published by the International Electrotechnical Commission. The library is written in Java and permits FLC design and implementation, following IEC standard for Fuzzy Control Language (FCL). The standard defines a common language to exchange portable fuzzy control programs among different platforms. The aim of jFuzzyLogic is to bring the benefits of open source software and standardization to the researchers and practitioners of fuzzy systems community, namely:

- Standardization, which reduces programming work.

- Extensibility, i.e., the object model and the Application Programming Interface (API) allow to create a wide range of different applications. 
- Platform independence, that permits the software development on whatever hardware platform and operating system supporting Java.

Moreover, jFuzzyLogic allows to implement a Fuzzy Inference System (FIS). A FIS is usually composed of one or more Function Blocks (FBs). Each FB has variables (input, output or instances) and one or more Rule Blocks (RBs). Each $\mathrm{RB}$ is composed of a set of rules, as well as Aggregation, Activation and Accumulation methods. Having said that, jFuzzyLogic is based on ANTLR [14 that generates a lexer and a parser based on a FCL grammar defined by the user. The parser, written in Java, uses a left to right leftmost derivation recursive strategy, formally known as $L L\left(^{*}\right)$. Using the lexer and the parser generated by ANTLR, it can parse FCL files by creating an Abstract Syntax Tree (AST), a well known structure in compiler design. The AST is converted into an Interpreter Syntax Tree (IST), which is capable of performing the required computations. This means that the IST can represent the grammar, in a similar way of AST, but it is also capable of performing computations. Moreover, all methods defined in the IEC norm are implemented in jFuzzyLogic. It should be noted that jFuzzyLogic fulfills the definitions of Aggregation, Activation and Accumulation as defined in [10. Aggregation methods define the t-norms and t-conorms correspond to the familiar AND, OR and NOT operators [3. These can be Minimum, Product or Bounded difference operators. Each set of operators fulfills De Morgan's laws [5]. The Activation method establishes how rule antecedents modify the consequent. Activation operators are Minimum and Product. Finally, accumulation methods determines the way the consequents from multiple rules in the same RB are combined. Accumulation methods defined in the IEC norm include: Maximum, Bounded sum, Normed sum, Probabilistic OR and Sum [10]. Only two membership functions are defined in the IEC standard, i.e., singleton and piece-wise linear. $\mathrm{jFuzzyLogic} \mathrm{implements} \mathrm{other} \mathrm{membership} \mathrm{functions} \mathrm{such}$ as trapezoidal, sigmoidal, gaussian, generalized bell, difference of sigmoidal, and cosine, too. Moreover, jFuzzyLogic permits making arbitrary memberships by means of a combination of mathematical functions. Thanks to the flexibility in defining membership functions that can be sampled in a fixed number of points, called samples. The number, that is by default one thousand, can be modified on the basis of the precision-speed trade-off required for a specific application. Inference is performed by evaluating membership functions at these samples. To perform the sampling, the domain, called universe, of each variable, must be estimated. The variable universe is defined as the range where the variable assumes non-negligible value. For each variable, both membership function and term are considered during the universe computation. After the examination of all rules is terminated, the accumulation for each variable is complete. The last step in the evaluation of a FIS is the defuzzification [12. The value for each variable is computed by means of the defuzzification method selected by the user within the following set: Center of gravity, Rightmost Max, Center of area, Leftmost Max, Mean max (for the continuous membership functions), or Center of gravity (for the discrete membership functions). 


\section{System Validation}

The FDSS, object of the work, has a knowledge base organized in $123 \mathrm{FBs}$ and it consists of 6215 rules of the type described in Section 3. FDSS was tested producing about 150 ERAs related to GM plants (e.g., Bt-maize1 and Brassica napus). The ERAs, yielded by FDSS, were submitted to a pool of ISPRA2 experts, not involved in the FDSS knowledge base definition, in order to assess the consistency and completeness of FDSS evaluations. For instance, we present two ERAs taken from the 150 ERAs produced by FDSS. The former estimates that all risks about GMP cultivation (see Tab 1, scenario 1) are negligible. Instead, the latter identify some risks that are potentially high (see Tab 1, scenario 2). FDSS has been developed as a Web Application and uses MySql database to store the data required to perform the ERA process. FDSS is implemented in Java2EE under Linux UBUNTU on a PC with Intel dual core $2.6 \mathrm{GHz}, 2 \mathrm{~GB}$ RAM. It requires a CPU time lower than 2 sec to perform the whole ERA.

Table 1. Estimation of the risks for two scenarios. Value is in the $[0,100]$ interval and LV are the Linguistic Variables.

\begin{tabular}{|c|cc|cc|}
\hline Risk & Scenario 1 & Scenario 2 & \\
value & LV & value & LV \\
\hline Molecular aspect & 0.0 & low & 50.0 & medium \\
Potential expression of the inserts & 50.0 & medium & 50.0 & medium \\
Potential risk of the insert & 0.0 & low & 0.0 & low \\
Potential risks of the sequences & 0.0 & low & 100.0 & high \\
Potential risk of toxicity & 0.0 & low & 0.0 & low \\
Surface involved & 0.0 & low & 100.0 & high \\
\hline
\end{tabular}

\section{Conclusions}

In this paper a FDSS for the ERA of the deliberate release of GMPs has been presented. The evaluation process permits identifying potential impacts that can achieve one or more receptors through a set of migration paths. The risk assessment in the FDSS is obtained by using a FIS, implemented by means of jFuzzyLogic library. The decisions yielded by FDSS have been validated on real world cases by the human experts of ISPRA, confirming, in this way, the FDSS reliability. In the next future we plan to develop machine learning algorithms that allow to learn automatically the knowledge base of FDSS.

Acknowledgements. This research has been partially funded by the LIFE project MAN-GMP-ITA (Agreement n. LIFE08 NAT/IT/000334).

${ }^{1}$ Maize modified by using a Bt toxin (Bacillus thuringensis) 16.

${ }^{2}$ ISPRA is the institute governed by the Italian Ministery of the Environment that is in charge of GMO risk estimation. 


\section{References}

1. Chen, Y.-L., Weng, C.-H.: Mining fuzzy association rules from questionnaire data. Knowledge-Based Systems 22, 46-56 (2009)

2. Chen, Z., Zhao, L., Lee, K.: Environmental risk assessment of offshore produced water discharges using a hybrid fuzzy-stochastic modeling approach. Environmental Modelling \& Software 25, 782-792 (2010)

3. Ciaramella, A., Tagliaferri, R., Pedrycz, W.: The genetic development of ordinal sums. Fuzzy Sets and Systems 151(2), 303-325 (2005)

4. Cingolani, P., Fdez, J.A.: jFuzzyLogic: A Robust and Flexible Fuzzy-Logic Inference System Language Implementation. In: Proceedings of IEEE World Congress on Computational Intelligence 2012 (2012)

5. Cormen, T.H., Leiserson, C.E., Rivest, R.L., Stein, C.: Introduction to Algorithms, 3rd edn. The MIT Press (2009)

6. Davidson, V.J., Ryks, J., Fazil, A.: Fuzzy risk assessment tool for microbial hazards in food systems. Fuzzy Sets and Systems 157, 1201-1210 (2006)

7. Guimara, A.C.F., Lapa, C.M.F.: Fuzzy inference to risk assessment on nuclear engineering systems. Applied Soft Computing 7, 17-28 (2007)

8. Kahraman, C., Kaya, I.: Fuzzy Process Accuracy Index to Evaluate Risk Assessment of Drought Effects in Turkey. Human and Ecological Risk Assessment 15, 789-810 (2009)

9. Karimi, I., Hullermeier, E.: Risk assessment system of natural hazards: A new approach based on fuzzy probability. Fuzzy Sets and Systems 158, 987-999 (2007)

10. International Electrotechnical Commission technical committee industrial process measurement and control2. IEC 61131 - Programmable Controllers. Part 7: Fuzzy Control Programming. IEC 2000 (2000)

11. Li, W., Zhou, J., Xie, K., Xiong, X.: Power System Risk Assessment Using a Hybrid Method of Fuzzy Set and Monte Carlo Simulation. IEEE Transactions on Power Systems 23(2) (2008)

12. Lin, C.-T., Lee, C.S.: Neural Fuzzy Systems: A Neuro-Fuzzy Synergism to Intelligent Systems. Prentice Hall (1996)

13. Ngai, E.W.T., Wat, F.K.T.: Design and development of a fuzzy expert system for hotel selection. Omega 31, 275-286 (2003)

14. Parr, T.J., Quong, R.W.: Software: Practice and Experience 25(7), 789-810 (1995)

15. Sadiqa, R., Husain, T.: A fuzzy-based methodology for an aggregative environmental risk assessment: A case study of drilling waste. Environmental Modelling \& Software 20, 33-46 (2005)

16. Sorlini, C., Buiatti, M., Burgio, G., Cellini, F., Giovannelli, V., Lener, M., Massari, G., Perrino, P., Selva, E., Spagnoletti, A., Staiano, G.: La valutazione del rischio ambientale dell' immissione deliberata nell' ambiente di organismi geneticamente modificati. Tech. Report (2003) (in Italian), http://bch.minambiente.it/EN/Biosafety/propmet.asp

17. Wang, Y.-M., Elhag, T.M.S.: An adaptive neuro-fuzzy inference system for bridge risk assessment. Expert Systems with Applications 34, 3099-3106 (2008) 